\title{
Proposta de isolamento para aplicação de diamino fluoreto de prata
}

\section{Proposal for isolation for the application of silver diamine fluoride}

\author{
Alice Lage Bueno ${ }^{1}$ \\ Camila Miranda Pimenta Camisassa Diniz ${ }^{2}$ \\ Mônica Moura de Faria GonÇALVES ${ }^{3}$ \\ Cláudia ValÉria de Sousa Resende PENIDO ${ }^{4}$
}

\begin{abstract}
RESUMO
A utilização de cariostático é uma técnica efetiva para a paralisação da cárie tanto em dentes decíduos quanto em permanentes. Apesar de todas suas qualidades e vantagens, um dos desafios desse produto é seu indesejável efeito antiestético, dependendo da superfície na qual é aplicado. Como um dos componentes desse medicamento é a prata, provoca manchas de tom acastanhado nos dentes nos quais é aplicado. No caso de contato acidental da substância com pele ou mucosa, o manchamento também pode ocorrer. Essas manchas são reversíveis e desaparecem aproximadamente após uma semana.

Objetivo: Para evitar essa intercorrência, neste trabalho será apresentada uma nova proposta de isolamento do campo de trabalho, com objetivo de proteção perioral, para a aplicação de Diamino Fluoreto de Prata (DFP).

Material e Métodos: Será apresentado um caso clínico realizado em uma criança de 9 anos de idade, com cáries interproximais em molares. A técnica desse isolamento será demonstrada detalhadamente, relatando os materiais utilizados e as vantagens perante a técnica convencional de aplicação do material. Conclusão: A técnica proposta é eficaz, de fácil realização, possui baixo custo, boa aceitação pelos pacientes, não é invasiva e diminui as intercorrências.
\end{abstract}

Palavras-chave: Cariostáticos. Cárie dentária. Odontopediatria. Compostos de prata.

\section{ABSTRACT}

The use of cariostatic agents is an effective technique to stop the cavity not only in primary teeth but also in permanent teeth. Despite all its qualities and advantages, one of the challenges of this product is its undesirable anti-aesthetic effect, depending on the surface on which it is applied. Because one of the components of this medicine is silver, it causes brownish stains on the teeth to which the product is applied. In case of accidental contact of the substance with the skin or the oral mucosa, staining might happen as well. These stains are reversible, and they disappear within a week.

Objective: To avoid this event, this paper presents a new proposal for isolation of the work field, aiming perioral protection, so Silver Fluoride Diamine (SFD) can be applied.

Material and Methods: A case performed on a nine-year-old child with interproximal cavities in molar teeth will be presented. The technique of this isolation will be shown in detail, reporting all the material used as well as the advantages over the conventional technique of application of this material.

Conclusion: The proposed technique is effective, easy to perform, has low cost, well accepted by the patients, is noninvasive and reduces all possible complications.

Keywords: Cariostatic agents. Dental caries. Pediatric dentistry. Silver compounds.

\section{Cirurgiã-dentista.}

Cirurgiã-dentista.

Professora Adjunta IV do Curso de Odontologia da Faculdade de Odontologia da Pontifícia Universidade Católica de Minas Gerais.

4 Professora Adjunta IV do Curso de Odontologia da Faculdade de Odontologia da Pontifícia Universidade Católica de Minas Gerais. 


\section{INTRODUÇÃO}

Para melhorar o atendimento das crianças em casos de cáries interproximais de esmalte, hoje é muito utilizada a aplicação de DFP. Este é um agente muito eficaz, tem baixo custo, é indolor, seguro, de fácil aplicação, dispensa a utilização de anestesia e brocas, não destrói a estrutura do dente e tem alta eficácia. Além disso, possui propriedades cariostáticas, anticariogênicas, preventivas, remineralizantes e dessensibilizantes. ${ }^{1-4}$ Esse material possui três mecanismos de ação, sendo o primeiro o da obstrução dos túbulos dentinários. A ação cariostática é o segundo mecanismo, que se dá por meio do produto da reação entre o DFP e o componente mineral do dente que aumenta a resistência à descalcificação ácida. O terceiro mecanismo é a ação enzimática, que se caracteriza pelo aumento da resistência da proteína dentinária. ${ }^{5}$

Apesar de todas suas qualidades e vantagens, um dos desafios desse produto é seu indesejável efeito antiestético. Como um dos componentes desse medicamento é a prata, sua precipitação e oxidação provocam manchas de tom acastanhado nos dentes nos quais é aplicado. ${ }^{2}$ No caso de contato acidental da substância com pele ou mucosa, o mesmo pode ocorrer ficando aparentes manchas semelhantes à tatuagem com henna. Estas são reversíveis e desaparecem após natural descamação dos tecidos, entre uma e duas semanas $^{5}$ (Fig. 1 (A) e (B)). Recentemente, o DFP tem sido indicado para tratamento de lesões interproximais. Nessa superfície não há essa desvantagem, não afetando a aceitação dos pais, fazendo assim que essa opção possa ser indicada em casos selecionados.

Sabendo que um bom isolamento ajuda na diminuição das intercorrências, ${ }^{6}$ o objetivo deste trabalho é apresentar um caso no qual foi empregada uma nova proposta de isolamento dos tecidos moles ao redor do dente e na região perioral para aplicação do DFP.
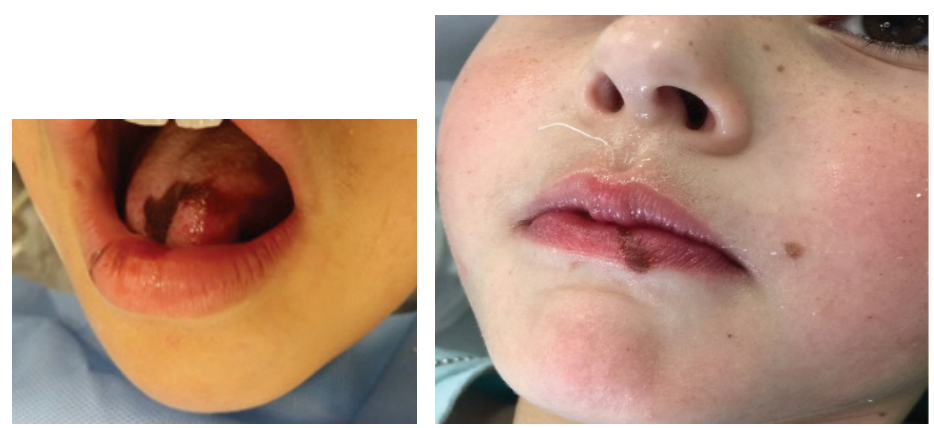

Fig. 1 (A) (B) - Exemplo de pigmentação de língua $(A)$ e lábios/ região perioral $(B)$ provocada por DFP.

\section{Relato de CASO}

Criança com 9 anos de idade, gênero masculino, acompanhada da sua mãe procurou atendimento odontológico. Ao exame clínico, foi observada má higienização oral e presença de lesão incipiente na superfície mesial do dente 75 , que estava visível no exame clínico, pois o dente 74 havia esfoliado e o permanente sucessor ainda não estava em oclusão. Ao exame radiográfico interproximal foi constatada lesão radiolúcida também na distal desse elemento (Fig. 2). Para esse dente, após analisadas as alternativas de tratamento, foi indicada a técnica de aplicação do DFP com isolamento relativo, associado à proteção dos tecidos periorais: lençol de borracha e expandex.

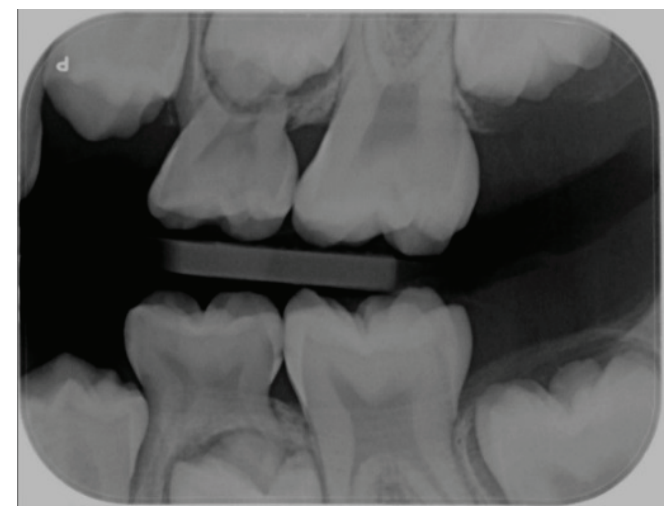

Fig. 2 - Exame radiográfico interproximal para diagnóstico das lesões.

Nessa técnica, além da utilização de isolamento relativo como convencionalmente é realizado, foi também empregado o uso de isolamento absoluto associado ao expandex com o objetivo de evitar o 
risco de pigmentação da pele e lábios do paciente. Essa nova técnica proposta está descrita a seguir. Inicialmente, foi aplicada vaselina na região perioral, logo após foi feito um corte reto no meio de um lençol de borracha de modo que ele não se partisse ao meio. Este foi colocado no expandex e levado à boca do paciente. Ao colocá-lo, foi certificado que todo o lábio e região peribucal estivessem sem risco de manchar. Rolinhos de algodão foram posicionados para afastar a língua e a mucosa jugal. O sugador foi acomodado e mantido durante todo o procedimento (Fig. $3(A)$ e (B).

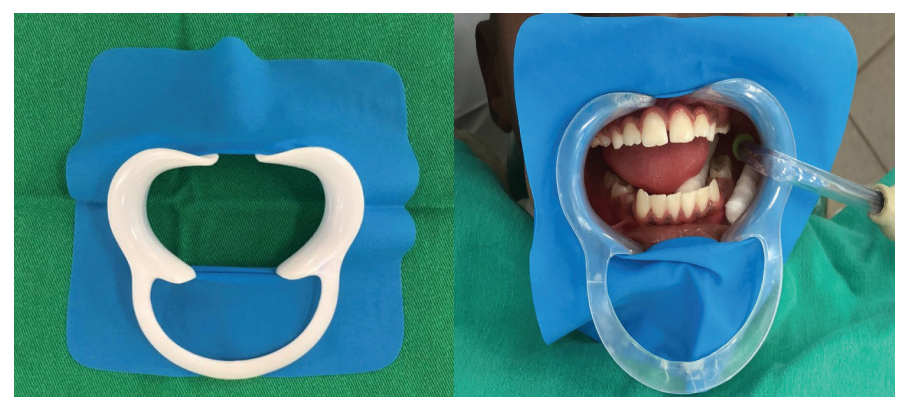

Fig. 3 (A) (B) - Lençol de borracha posicionado no expandex observar corte horizontal.

A partir desse momento, seguiu-se a técnica normal de aplicação do cariostático, colocando o superfloss (parte com espuma) entre os dentes 75 e 36. Molhou-se o microbrush com DFP e este foi levado ao fio dental por vestibular e lingual, alternada e repetidamente, sempre bem próximo ao dente até encharcar a espuma. Quando a espuma estava bem encharcada, dispensou-se o microbrush e movimentos curtos e suaves com o fio foram realizados durante 1 minuto (Fig. 4). Na mesial do dente 75 , foi feita a aplicação direta com microbrush devido à ausência do dente 74. Secouse o local com jato de ar e se removeu o excesso do produto com gaze. ${ }^{1}$ Depois, retirou-se o fio tomando cuidado para não esbarrar em mucosa e língua e em seguida os rolinhos de algodão. Após, o conjunto expandex e o lençol de borracha foram retirados, a luva do operador foi trocada e, então, o rosto da criança foi limpo.

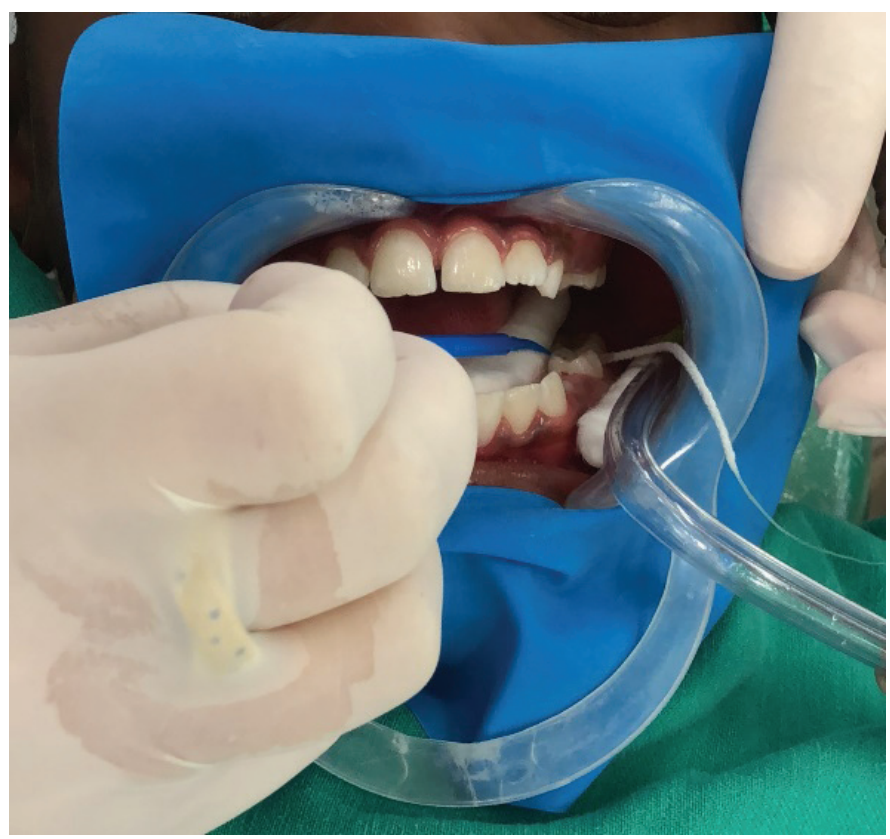

Fig. 4- Aplicação do diamino com superfloss.

\section{Discussão}

Quando há diagnóstico de lesões interproximais incipientes, estas podem ser revertidas com o uso e métodos caseiros como: uso de fio dental, pasta fluoretada e bochechos fluoretados, associados com aplicação profissional de fluoretos. Porém, esses métodos dependem da adesão do paciente e da família. ${ }^{7}$ Nessas situações, o uso de DFP é uma alternativa viável por ser um produto bastante efetivo, no que concerne a controlar e paralisar a doença cárie, devido à suas propriedades preventivas e cariostáticas, sendo esse método de baixo custo e fácil aplicação. Em função de sua técnica de aplicação simples e seus bons resultados, essa alternativa de tratamento está sendo considerada por muitos profissionais, principalmente na área de Odontopediatria, ${ }^{3,8}$ como parte de programa de manejo de lesões de cárie..$^{9,10}$ Esse método está relacionado entre as técnicas recomendadas pela Associação Americana de Odontopediatria, que disponibiliza o protocolo de uso do mesmo. ${ }^{1,11}$ É importante ressaltar que a prevenção de novas lesões deve envolver todo o núcleo familiar, fazendo parte das estratégias a serem utilizadas pelo cirurgião- 
dentista para que se obtenha sucesso no tratamento.

Sua ação é capaz de aumentar a resistência do esmalte ao processo de desmineralizaçãoremineralização, e de promover um efeito antibacteriano. ${ }^{2,4}$ Porém, uma das maiores desvantagens dessa solução é a possibilidade de escurecimento da pele ou mucosa perioral causado pela precipitação dos íons prata em contato acidental com essas regiões. ${ }^{5}$ Essa nova proposta de isolamento para a aplicação da solução do DFP tem o objetivo de evitar que essas manchas escurecidas aconteçam acidentalmente durante o procedimento, de maneira prática e atraumática. ${ }^{12}$ Outra vantagem é a não utilização de grampos, que remetem à não necessidade de anestesias, assim como para a aplicação da solução que não utiliza caneta de alta rotação e brocas, ainda com o intuito de permitir um maior conforto para o paciente..$^{3,6}$

Um dos grandes desafios do uso de DFP são os critérios de indicação do mesmo e o risco de pigmentação de dentes, por isso atualmente há a tendência de utilizá-lo somente em superfícies que não interferem na estética. Outro é também a pigmentação da pele e lábios. Tudo isso ressalta a importância da proposta deste trabalho. $\mathrm{O}$ isolamento é confortável para o paciente, indolor, seguro, de fácil aplicação e bastante eficaz quanto ao seu objetivo de isolar a região que vai receber a solução cariostática e para prevenir que as superfícies periorais possam manchar caso ocorra algum contato acidental. Como o DFP é manipulado pelo operador durante a aplicação, este deve ter o cuidado de trocar as luvas ao final do procedimento, mesmo ao fazer aplicações em outros hemi-arcos do mesmo paciente, antes da limpeza do rosto do paciente, para que não ocorra qualquer alteração no paciente.

\section{CONSIDERAÇÕES FINAIS}

A técnica proposta é eficaz, de fácil realização, possui baixo custo, boa aceitação pelos pacientes, não é invasiva e diminui as intercorrências.

\section{REFERÊNCIAS}

1. American Academy of Pediatric Dentistry. Use of silver diamine fluoride for dental caries management in children and adolescents, including those with special health care needs. In: American Academy of Pediatric Dentistry. Recommendations: clinical practice guidelines: reference manual. Chicago: AAPD; 2017, p. 152-61.

https://www.aapd.org/research/oral-health-policies--recommendations/silver-diamine-fluoride-for-dental-caries-management-in-children-and-adolescents-including-those-with-special-health-care-needs/

2. Triches TC, Cordeiro MMR, Souza JGMV, Saltori EK, França, BHS. Aceitação dos pais quanto ao uso do diamino fluoreto de prata em crianças de 0 a 3 anos de Cascavel/PR. Pesqui Bras Odontopediatria Clin Integr 2009; 9 (3): 265-9. http://revista.uepb.edu.br/index.php/ pboci/article/viewFile/734/388

3. Ditterich RG, Romanelli MCMOV, Rastelli MC, Czlusniak GD, Wambier DS. Fluoreto de prata: uma revisão de literatura. Publ UEPG Ci Biol Saúde 2006; 12 (2): 45-52.

http://www.revistas2.uepg.br/index.php/biologica/article/view/433

4. Gao SS, Zhao IS, Hiraishi N., Duandthip D., Mei ML, Lo ECM, et al. Clinical trials of silver diamine fluoride in arresting caries among children: A systematic review. JDR Clin Trans

Res. 2016; 1 (3): 201-10.

https://journals.sagepub.com/doi/ pdf/10.1177/2380084416661474

5. Santos Junior VE, Souza PR, Rosenblatt $A$. Um recurso para paralisar e prevenir cárie em crianças: Diamino Fluoreto de Prata. RFO UPF 2012; 17 (2): 228-33.

http://revodonto.bvsalud.org/scielo.php?pi 
$d=S 141340122012000200019 \&$ script $=$ sci arttext\&tlng=pt

6. Gordon NB. Silver diamine fluoride staining is acceptable for posterior primary teeth and is preferred over advanced pharmacologic behavior management by many parents. J Evid Base Dent Pract 2018; 18 (1): 94-97.

https://www.sciencedirect.com/science/article/ abs/pii/S1532338218300058

7. Vargas AN, Fontes BNV, Tolentino AB, Rodrigues LCM, Oliveira FS, Castro AM. Percepção do estudante de Odontologia sobre os fatores estressores relacionados ao atendimento infantil. Rev Fac Odontol Lins 2013; 23 (1): 11-9.

https://www.metodista.br/revistas/revistas-unimep/index.php/Fol/article/view/1756/0

8. Gold J. Silver diamine fluoride arrest caries in primary teeth. J Evid Base Dent Pract 2018; 18 (1): 88-90.

https://www.sciencedirect.com/science/article/ abs/pii/S1532338217303901

9. Mattos-Silveira J., Floriano I., Ferreira FR, Viganó ME, Frizzo MA, Reyes A., et al. New proposal of silver diamine fluoride use in arresting approximal caries: study protocol for a random- ized controlled trial. Trials 2014; 15: 448.

https://www.ncbi.nlm.nih.gov/pmc/articles/ PMC4255679/

10. Huebner CE, Milgron P., Cunha-Cruz J., Scott J., Spiekerman C., Ludwig S., et al. Parent's Satisfaction with Silver Diamine Fluoride Treatment of Carious Lesions in Children. J Dent

Child 2020; 87 (1): 4-11.

https://pesquisa.bvsalud.org/controlecancer/resource/pt/mdl-32151304

11. Pollick $\mathrm{H}$. The role of fluoride in the prevention of tooth decay. Pediatr Clin North America 2018; 65 (5): 923-40.

https://www.researchgate.net/publication/327732309_The_Role_of_Fluoride_in_ the_Prevention_of_Tooth_Decay

12. Sousa FSO, Santos APP, Barja-Fidalgo F., Oliveira $\mathrm{BH}$. Evidence-based pediatric dental practice within the clinician's reach: the case of the esthetic effect of topical silver diamine fluoride for caries control in primary dentition. RGO 2016; 64 (4): 369-75.

http://pesquisa.bvsalud.org/portal/resource/pt/ biblio-842337

Submetido em: $14-8-2020$

Aceito em: 26-11-2020 